Article

\title{
Longitudinal Event-Level Sexual Risk and Substance Use among Gay, Bisexual, and Other Men Who Have Sex with Men
}

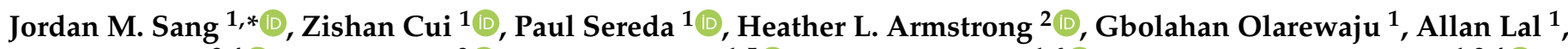

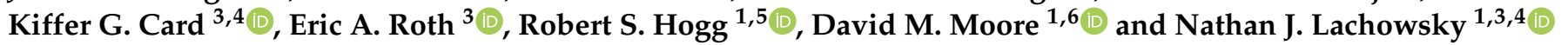 \\ 1 British Columbia Centre for Excellence in HIV / AIDS, Vancouver, BC V6Z 1Y6, Canada; \\ cuizishan@gmail.com (Z.C.); psereda@bccfe.ca (P.S.); golarewa@gmail.com (G.O.); alal@bccfe.ca (A.L.); \\ bobhogg@bccfe.ca or robert_hogg@sfu.ca (R.S.H.); dmoore@bccfe.ca (D.M.M.); nlachowsky@uvic.ca (N.J.L.) \\ 2 School of Psychology, University of Southhampton, Southampton SO17 1BJ, UK; H.Armstrong@soton.ac.uk \\ 3 School of Public Health and Social Policy, University of Victoria, Victoria, BC V8P 5C2, Canada; \\ kiffercard@gmail.com (K.G.C.); ericroth@uvic.ca (E.A.R.) \\ 4 Canadian Institute for Substance Use Research, University of Victoria, Victoria, BC V8P 5C2, Canada \\ 5 Faculty of Health Sciences, Simon Fraser University, Burnaby, BC V5A 1S6, Canada \\ 6 Department of Medicine, Faculty of Medicine, University of British Columbia, \\ Vancouver, BC V6T 1Z4, Canada \\ * Correspondence: jsang@bccfe.ca
}

check for

updates

Citation: Sang, J.M.; Cui, Z.; Sereda, P.; Armstrong, H.L.; Olarewaju, G.;

Lal, A.; Card, K.G.; Roth, E.A.; Hogg, R.S.; Moore, D.M.; et al. Longitudinal Event-Level Sexual Risk and

Substance Use among Gay, Bisexual, and Other Men Who Have Sex with Men. Int. J. Environ. Res. Public Health 2021, 18, 3183. https://doi.org/ 10.3390/ijerph18063183

Academic Editor: Paul B. Tchounwou

Received: 20 January 2021

Accepted: 16 March 2021

Published: 19 March 2021

Publisher's Note: MDPI stays neutral with regard to jurisdictional claims in published maps and institutional affiliations.

Copyright: (c) 2021 by the authors. Licensee MDPI, Basel, Switzerland. This article is an open access article distributed under the terms and conditions of the Creative Commons Attribution (CC BY) license (https:// creativecommons.org/licenses/by/ $4.0 /)$.
Abstract: (1) Background: Condomless anal sex and substance use are associated with STI risk among gay, bisexual, and other men who have sex with men (gbMSM). Our first study objective was to describe event-level sexual risk and substance use trends among gbMSM. Our second study objective was to describe substances associated with event-level sexual risk. (2) Methods: Data come from the Momentum Health Study in Vancouver, British Columbia and participants were recruited from 2012-2015, with follow-up until 2018. Stratified by self-reported HIV status, we used generalized estimating equations to assess trends of sexual event-level substance use and assessed interactions between substance use and time period on event-level higher risk sex defined as condomless anal sex with an HIV serodifferent or unknown status partner. (3) Results: Event-level higher risk anal sex increased across the study period among HIV-negative/unknown (baseline prevalence: 13\% vs. study end prevalence: $29 \%$ ) and HIV-positive gbMSM (baseline prevalence: $16 \%$ vs. study end prevalence: 38\%). Among HIV-negative/unknown gbMSM, event-level erectile drug use increased, while alcohol use decreased over the study period. Overall, interactions between substance use and time on higher risk anal sex were not statistically significant, regardless of serostatus. However, we found a number of time-specific significant interactions for erectile drugs, poppers, Gamma-hydroxybutyrate (GHB), crystal methamphetamine and ecstasy/MDMA use among HIV-negative/unknown gbMSM. (4) Conclusion: Significant differences in substance use trends and associated risks exist and are varied among gbMSM by serostatus. These findings provide a more comprehensive understanding of the effects of event-level substance use on sexual risk through longitudinal follow-up of nearly six years.

Keywords: sexual risk; trends; event-level; substance use; MSM; STI

\section{Introduction}

Rates of sexually transmitted infections (STI) are rising in Canada and are highly concentrated among gay, bisexual, and other men who have sex with men (gbMSM) [1]. Further, disparities in STI prevalence, including diagnoses of chlamydia, gonorrhea, syphilis, and lymphogranuloma venereum (LGV), suggest HIV serostatus plays an important role in STI transmission [1,2]. Serosorting is a common practice among gbMSM, where individuals select sexual partners based on HIV status. However, serosorting based on HIV status may increase rates of STIs as gbMSM may forgo condoms with the same HIV serostatus partners, concentrating STI rates among HIV-positive gbMSM [3]. Moreover, higher rates 
of serodiscordant condomless anal sex (CAS) are reported among HIV-positive gbMSM compared to HIV-negative gbMSM [4], with serodiscordant CAS identified as the main factor in STI diagnoses among HIV-positive among gbMSM [5]. Within Canada, a study of gbMSM living with HIV found an increased risk of chlamydia and gonorrhea was associated with multiple HIV-positive partners as well as recreational drug use [6]. Collectively, STI's may be more prevalent among certain sexual networks of gbMSM and considerations of HIV serostatus on STI risk are important to further understand sexual risk.

Substance use is a significant factor in influencing sexual risk-taking behaviors, such as CAS. Previous literature on substance use patterns among gbMSM indicates variability in different substance types and distinct classes of substances, such as club drug use, sex drug use and conventional drug use [7]. A systematic review on sexualized drug use among gbMSM found multiple factors that may promote engagement in sexualized drug use, such as coping with stressful events, increasing intimacy, fulfilling community belonging and enhancing sexual performance and functioning [8]. Related, chemsex refers to certain recreational drugs (particularly combinations of crystal methamphetamine, mephedrone and gamma-hydroxybutyrate/gamma-butyrolactone (GHB/GBL)) used before or during sex, which help facilitate or enhance sex [9]. Among gbMSM living with HIV, multiple studies have found chemsex significantly associated with serodiscordant CAS, serodiscordant CAS with a partner, who has a detectable viral load, increased number of sexual partners, and increased STI diagnoses [10,11]. Among HIV-negative gbMSM, existing literature found chemsex was associated with serodiscordant/HIV-unknown CAS, STI diagnoses, a greater number of sexual partners and group sex events [12]. Mixed-method research from the United Kingdom help contextualize findings as gbMSM reports difficulty negotiating safer sex while under the influence of substances, and perceptions about HIV and STI sexual risk may also be skewed [13].

The association between substance use and sexual risk relates to situational events, which are highly contextual and may vary in terms of substances used, partner characteristics, condom usage, and sexual acts. To address these limitations, various event-level approaches focusing on specific sexual encounters and substance use within highly restricted time periods before or during sex may provide nuanced understanding [14]. A review of the literature on event-level substance use and sexual behaviors among gbMSM found consistent associations of sexual risk with methamphetamine use and alcohol binge drinking among gbMSM [15]. However, inconsistencies in event-level measurement and analysis may limit generalizability in findings. For example, using both retrospective and prospective event-level data, Rendina et al. (2015) found event-level substance use, in particular, club drugs, such as ketamine, ecstasy/3,4-methylenedioxymethamphetamine (MDMA), GHB, cocaine/crack, or methamphetamine, increased odds of sex and engaging in CAS [16]. Among gbMSM living with HIV, Sullivan and colleagues found self-reported heavier alcohol users reported less frequent condom use [17]. Associations between substance use and CAS are also not limited by age, as consistent event-level diary findings among young gbMSM also found associations between alcohol use and CAS with casual partners [18]. While event-level substance use has been associated with CAS among gbMSM, overall event-level substance use has not been found to be associated with perceived control or pleasure during sex [19]. However, individual substances such as crystal methamphetamine use has been found to be significant for both CAS and lower perceived control during sex [19]. Taken together, various event-level approaches highlight evidence for associations between substance use and sexual risk. However, research within a Canadian context is limited.

Using event-level longitudinal data to measure STI sexual risk, our first study objective was to describe event-level sexual risk and substance use trends in Vancouver during treatment as prevention scale-up (TasP) over a six-year period [20]. Our second objective was to describe individual and partner substance use associated with event-level sexual risk and to determine whether there have been any changes in the significance of certain substances as they relate to condom use over the study period. 


\section{Materials and Methods}

\subsection{Study Protocol and Participants}

Data are from the Momentum Health Study, a prospective longitudinal, bio-behavioral study of gbMSM in Vancouver, British Columbia. Participants were recruited from February 2012 to February 2015 using respondent-driven sampling (RDS). RDS recruitment involved initial "seed" participants, who were recruited through community partner agencies and online advertisements on gbMSM social networking websites and apps. The full RDS methodology of our study has been published elsewhere [21]. To be eligible, participants had to gender-identify as a man, be 16 years of age or older, report having sex with another man in the past six months, currently live in Metro Vancouver, and be able to complete the questionnaire in English. Participants completed a $90 \mathrm{~min}$ in-person study visit every six months, which included a computer-assisted self-interview (CASI) and study nurse visit. Participants received a \$50 CAD honorarium for their participation and could receive an additional \$10 CAD for each eligible participant they referred that completed the study (maximum of six). Study visits up to February 2018 are included in this analysis. All participants signed an Informed Consent form about the study and their involvement. The research protocol and human ethics clearances were approved by The University of British Columbia, Simon Fraser University, and The University of Victoria.

\subsection{Outcome Variable}

The primary outcome variable was higher risk anal sex, which was defined as any CAS with an HIV serodifferent or unknown status partner. Participants were asked to complete a "partner matrix" of a repeating set of questions about their last sexual encounter with each of up to five of their most recent sexual partners within the past six months (maximum of five partners). Sexual encounters that did not include anal sex were excluded in this analysis. Condom use was reported for each partner, and participants indicated their use/non-use as the receptive and insertive partner. Any form of CAS (receptive or insertive) was included in this analysis. The partner's HIV serostatus was obtained by asking participants if they knew their partner(s)'s HIV status before having sex, what the partner's status was, and how they knew their partner's status (if they knew). From these, we determined if the partner's serostatus was positive, negative, or unknown.

\subsection{Explanatory Variables}

The primary explanatory factors were time (for trend analyses) and substance use. Time of event was assessed with a six-month period prevalence between study visits over the course of almost six years. Event-level factors were collected for each partner and reported sexual event. Participants indicated the number of male sexual partners, the number of months since they first had sex with each partner, and the number of times they had anal sex with each partner in the past six months (per act). Participants indicated the month and year of the last sexual event with each partner, which was used to conduct a change over time analysis. For each sexual event, participants indicated their anal sex positions (receptive, insertive, or both), their level of certainty regarding their partner's HIV status before sex, whether they expected they would have sex with this partner again, and whether they received any goods, money, drugs, or services in return for sex. Participants reported their own and their partner's substance use in the two hours prior to and during each sexual event, which included any alcohol, cannabis, erectile drugs, poppers (amyl nitrate), crystal methamphetamine, GHB, and MDMA.

Psychosocial variables included the HIV treatment optimism-skepticism scale (12 questions, range: $12-48$, study $\alpha=0.85$ ) [22], the 11-item sexual seeking scale (revised) (range: $11-44$, study $\alpha=0.73$ ) [23], the 7-item personal (range: $1-5, \alpha=0.75$ ) and 6-item communal subscales for the sexual altruism scale (range: $1-5$, study $\alpha=0.77$ ) [24], and the 10-item alcohol use disorders identification test (AUDIT) (range: 0-40, study $\alpha=0.86$ ) [25].

Demographic variables included participants' age, sexual orientation, race/ethnicity, annual income, education, residence, and relationship status. We also asked a series of 
potential HIV prevention or risk-reduction practices (i.e., always using condoms, seropositioning, serosorting, viral-load sorting, abstinence, withdrawal, asking for HIV status before sex), PrEP usage, escort work and attending group sex events in the past six months. For HIV-positive gbMSM, we utilized the study linkage to the BC Centre for Excellence in HIV/AIDS's Drug Treatment Program administrative database to assess treatment adherence and viral load [20].

\subsection{Analysis}

We limited our analyses to the sexual-event level and stratified participants by selfreported HIV status. Generalized estimating equations (GEE) were used to construct hierarchical logistic regression models, adjusting for participant interdependence in the data (events within participants as main clusters and each visit as sub-clusters). We examined trends over time with higher risk anal sex and substance use. Furthermore, we also tested interactions for substances and time to assess whether their associations with higher risk anal sex significantly changed over the study period. Odds ratio per six-months are presented, and significance was assessed as a $p$-value $<0.05$. RDS weighting was not applied, given that the analysis is based on event-level data. We included post hoc lostto-follow-up analyses (LTFU) in determining significant differences between participants who did not complete the study and our final sample. All analyses were conducted using SAS version 9.4 (SAS, Cary, NC, USA).

\section{Results}

\subsection{Descriptive Results}

The median follow-up time for participants was 3.03 years. 549 HIV-negative/ unknown gbMSM reported 8121 anal sexual events, of which $17.9 \%$ included CAS with a serodifferent or unknown status partner. Among the 213 HIV-positive gbMSM at baseline, 3454 anal sexual events were reported, of which $27.9 \%$ included CAS with a serodifferent or unknown status partner. Full descriptive statistics on the sample stratified by HIV status can be found in Table 1.

Table 1. Baseline participant-level descriptors of gbMSM in Metro Vancouver, stratified by selfreported HIV status.

\begin{tabular}{|c|c|c|c|c|c|c|}
\hline \multirow[t]{2}{*}{ Variables } & \multirow{2}{*}{\multicolumn{2}{|c|}{$\begin{array}{c}\text { Overall } \\
N\end{array}$}} & \multicolumn{2}{|c|}{ HIV-Negative/Unknown } & \multicolumn{2}{|c|}{ HIV-Positive } \\
\hline & & & $n$ & $\%$ & $n$ & $\%$ \\
\hline & \multicolumn{2}{|c|}{762} & 549 & 72 & 213 & 28 \\
\hline Age & & & & & & \\
\hline $16-29$ & 288 & 37.8 & 277 & 50.5 & 11 & 5.2 \\
\hline $30-39$ & 254 & 33.3 & 180 & 32.8 & 74 & 34.7 \\
\hline $40+$ & 220 & 28.9 & 92 & 16.8 & 128 & 60.1 \\
\hline \multicolumn{7}{|l|}{ Sexual Orientation } \\
\hline Gay & 647 & 84.9 & 464 & 84.5 & 183 & 85.9 \\
\hline Bisexual & 69 & 9.1 & 50 & 9.1 & 19 & 8.9 \\
\hline Other & 46 & 6.0 & 35 & 6.4 & 11 & 5.2 \\
\hline \multicolumn{7}{|l|}{ Ethnicity } \\
\hline White & 577 & 75.7 & 408 & 74.3 & 169 & 79.3 \\
\hline Asian & 74 & 9.7 & 62 & 11.3 & 12 & 5.6 \\
\hline Indigenous & 46 & 6.0 & 28 & 5.1 & 18 & 8.5 \\
\hline Latino & 35 & 4.6 & 28 & 5.1 & 7 & 3.3 \\
\hline Other & 30 & 3.9 & 23 & 4.2 & 7 & 3.3 \\
\hline \multicolumn{7}{|l|}{ Born in Canada } \\
\hline No & 176 & 23.1 & 140 & 25.5 & 36 & 16.9 \\
\hline Yes & 586 & 76.9 & 409 & 74.5 & 177 & 83.1 \\
\hline \multicolumn{7}{|l|}{ Neighborhood } \\
\hline Downtown/West End & 375 & 49.2 & 234 & 42.6 & 141 & 66.2 \\
\hline Elsewhere Vancouver & 238 & 31.2 & 192 & 35.0 & 46 & 21.6 \\
\hline Outside Vancouver & 149 & 19.6 & 123 & 22.4 & 26 & 12.2 \\
\hline \multicolumn{7}{|l|}{ Formal Education } \\
\hline Some or completed high school & 170 & 22.3 & 109 & 19.9 & 61 & 28.6 \\
\hline Any post-secondary training & 592 & 77.7 & 440 & 80.2 & 152 & 71.4 \\
\hline \multicolumn{7}{|l|}{ Annual Income } \\
\hline$<\$ 30,000$ & 476 & 62.5 & 324 & 59.0 & 152 & 71.4 \\
\hline at least $\$ 30,000$ & 286 & 37.5 & 225 & 41.0 & 61 & 28.6 \\
\hline
\end{tabular}


Table 1. Cont.

\begin{tabular}{|c|c|c|c|c|c|c|}
\hline \multirow[t]{2}{*}{ Variables } & \multirow{2}{*}{\multicolumn{2}{|c|}{$\begin{array}{c}\text { Overall } \\
N\end{array}$}} & \multicolumn{2}{|c|}{ HIV-Negative/Unknown } & \multicolumn{2}{|c|}{ HIV-Positive } \\
\hline & & & $n$ & $\%$ & $n$ & $\%$ \\
\hline & \multicolumn{2}{|c|}{762} & 549 & 72 & 213 & 28 \\
\hline \multicolumn{7}{|l|}{ Current Regular Partner } \\
\hline No & 470 & 61.7 & 337 & 61.4 & 133 & 62.4 \\
\hline Yes, but not common law/married & 163 & 21.4 & 120 & 21.9 & 43 & 20.2 \\
\hline Yes, common law/married & 129 & 16.9 & 92 & 16.8 & 37 & 17.4 \\
\hline \multicolumn{7}{|l|}{ Usage of PrEP } \\
\hline Yes & $\begin{array}{c}141 \\
1\end{array}$ & 0.2 & 0 & $\begin{array}{l}19.0 \\
0.0\end{array}$ & $\begin{array}{c}56 \\
1\end{array}$ & 0.7 \\
\hline Never heard of PrEP & 453 & 76.1 & 363 & 81.0 & $\begin{array}{l}1 \\
90\end{array}$ & 61.2 \\
\hline \multicolumn{7}{|l|}{ Escort Work } \\
\hline No & 625 & 82.0 & 481 & 87.6 & 144 & 67.6 \\
\hline Yes, in P6M & 47 & 6.2 & 30 & 5.5 & 17 & 8.0 \\
\hline & 90 & 11.8 & 38 & 6.9 & 52 & 24.4 \\
\hline \multicolumn{7}{|l|}{ Attended Group Sex P6M } \\
\hline Yes & 197 & 25.9 & 122 & 22.2 & 75 & 35.2 \\
\hline AUDIT Zone & & & & & & \\
\hline Low-risk (scores 0 to 7 ) & 455 & 60.0 & 293 & 53.5 & 162 & 76.8 \\
\hline Medium-risk (scores 8 to 15$)$ & 203 & 26.8 & 170 & 31.0 & 33 & 15.6 \\
\hline Harmful (scores 16 to 19$)$ & 54 & 7.1 & 45 & 8.2 & 9 & 4.3 \\
\hline $\begin{array}{c}\text { Possible dependence (scores } 20 \text { and } \\
\text { over) }\end{array}$ & 47 & 6.2 & 40 & 7.3 & 7 & 3.3 \\
\hline On ART Ever & & & & & & \\
\hline No & 3 & 1.4 & & & 3 & 1.4 \\
\hline Yes & 213 & 98.6 & & & 210 & 98.6 \\
\hline Treatment Adherence P12M & & & & & & \\
\hline $95 \%$ or greater & 120 & 55.6 & & & 120 & 56.3 \\
\hline $\begin{array}{c}<95 \% \\
\text { Sever }\end{array}$ & 53 & 24.5 & & & 53 & 24.9 \\
\hline Never on ART/start within $12 \mathrm{M}$ & 43 & 19.9 & & & 40 & 18.8 \\
\hline $\begin{array}{c}\text { Latest Viral Load <200 copies / mL } \\
\text { No }\end{array}$ & 37 & 17.1 & & & 34 & 16.0 \\
\hline Yes & 179 & 82.9 & & & 179 & 84.0 \\
\hline Prevention Strategies & & & & & & \\
\hline Always Using Condoms & & & & & & \\
\hline No & 334 & 44.1 & 190 & 34.9 & 144 & 67.6 \\
\hline Yes & 424 & 55.9 & 355 & 65.1 & 69 & 32.4 \\
\hline Seropositioning & & & & & & \\
\hline No & 539 & 71.1 & 400 & 73.4 & 139 & 65.3 \\
\hline Yes & 219 & 28.9 & 145 & 26.6 & 74 & 34.7 \\
\hline No Anal Sex & & & & & & \\
\hline No & 411 & 54.2 & 275 & 50.5 & 136 & 63.9 \\
\hline Yes & 347 & 45.8 & 270 & 49.5 & 77 & 36.2 \\
\hline Serosorting & & & & & & \\
\hline No & 453 & 59.8 & 352 & 64.6 & 101 & 47.4 \\
\hline Yes & 305 & 40.2 & 193 & 35.4 & 112 & 52.6 \\
\hline Viral-Load Sorting & & & & & & \\
\hline No & 617 & 81.4 & 490 & 89.9 & 127 & 59.6 \\
\hline Yes & 141 & 18.6 & 55 & 10.1 & 86 & 40.4 \\
\hline Withdrawal & & & & & & \\
\hline No & 538 & 71.0 & 392 & 71.9 & 146 & 68.5 \\
\hline Yes & 220 & 29.0 & 153 & 28.1 & 67 & 31.5 \\
\hline Asking Status & & & & & & \\
\hline No & 314 & 41.4 & 211 & 38.7 & 103 & 48.4 \\
\hline Yes & 444 & 58.6 & 334 & 61.3 & 110 & 51.6 \\
\hline Event-Level Outcomes & $n$ & $\%$ & $n$ & $\%$ & $n$ & $\%$ \\
\hline Higher risk anal sex & & & & & & \\
\hline No & 606 & 79.7 & 453 & 82.5 & 153 & 72.5 \\
\hline Yes & 154 & 20.3 & 96 & 17.5 & 58 & 27.5 \\
\hline Alcohol & & & & & & \\
\hline No & 434 & 57.0 & 295 & 53.7 & 139 & 65.3 \\
\hline Yes & 328 & 43.0 & 254 & 46.3 & 74 & 34.7 \\
\hline Cannabis & & & & & & \\
\hline No & 515 & 67.6 & 401 & 73.0 & 114 & 53.5 \\
\hline Yes & 247 & 32.4 & 148 & 27.0 & 99 & 46.5 \\
\hline Erectile Drugs & & & & & & \\
\hline No & 663 & 87.0 & 497 & 90.5 & 166 & 77.9 \\
\hline Yes & 99 & 13.0 & 52 & 9.5 & 47 & 22.1 \\
\hline Poppers & & & & & & \\
\hline No & 598 & 78.5 & 466 & 84.9 & 132 & 62.0 \\
\hline Yes & 164 & 21.5 & 83 & 15.1 & 81 & 38.0 \\
\hline Ecstasy/MDMA & & & & & & \\
\hline No & 723 & 94.9 & 524 & 95.5 & 199 & 93.4 \\
\hline Yes & 39 & 5.1 & 25 & 4.6 & 14 & 6.6 \\
\hline GHB & & & & & & \\
\hline No & 720 & 94.5 & 532 & 96.9 & 188 & 88.3 \\
\hline Yes & 42 & 5.5 & 17 & 3.1 & 25 & 11.7 \\
\hline
\end{tabular}


Table 1. Cont.

\begin{tabular}{ccccccc}
\hline Variables & \multicolumn{2}{c}{ Overall } & \multicolumn{2}{c}{ HIV-Negative/Unknown } & \multicolumn{2}{c}{ HIV-Positive } \\
\hline & \multicolumn{2}{c}{$\boldsymbol{N}$} & $\boldsymbol{n}$ & $\mathbf{\%}$ & $\boldsymbol{n}$ & $\mathbf{\%}$ \\
\hline & \multicolumn{2}{c}{$\mathbf{7 6 2}$} & $\mathbf{5 4 9}$ & $\mathbf{7 2}$ & $\mathbf{2 1 3}$ & $\mathbf{2 8}$ \\
\hline Crystal Methamphetamine & & & & & & \\
No & 685 & 89.9 & 520 & 94.7 & 165 & 77.5 \\
Yes & 77 & 10.1 & 29 & 5.3 & 48 & 22.5 \\
\hline Continuous Variables & Median & Q1, Q3 & Median & Q1, Q3 & Median & Q1, Q3 \\
\hline Male sex events number P6M & 4 & 1,15 & 4 & 2,20 & 3.5 & 1,12 \\
Anal sex events number P6M & 2 & 0,8 & 2 & 0,9 & 2 & 1,8 \\
Treatment optimism-skepticism scale & 25 & 21,28 & 24 & 20,27 & 28 & 25,32 \\
Sexual altruism scale (communal) & 3.5 & $2.8,4$ & 3.5 & 3,4 & 3.2 & $2.5,4$ \\
Sexual altruism scale (personal) & 3.4 & $3,3.9$ & 3.6 & $3.1,3.9$ & 3.3 & $2.7,3.9$ \\
Sexual sensation seeking scale & 31 & 28,34 & 30 & 28,33 & 32 & 29,35 \\
\hline
\end{tabular}

Notes: P6M = Past six months; P12M = Past 12 months; PrEP = Pre-exposure prophlaxis; ART = Antiretroviral Therapy; AUDIT = Alcohol Use Disorders Identification Test; MDMA = 3,4-methylenedioxymethamphetamine; $\mathrm{GHB}=3,4$-methylenedioxymethamphetamine.

\subsection{Analytical Results}

In our GEE, higher risk anal sex was significantly associated with greater use of poppers, erectile drug use, ecstasy/MDMA use, GHB use, and crystal methamphetamine use among HIV-negative/unknown gbMSM; only poppers use was significantly associated with greater odds of higher risk anal sex for HIV-positive gbMSM. Full results can be found in Table 2.

Table 2. Univariable generalized estimating equations assessing higher risk anal sex among gbMSM in Metro Vancouver, 2012-2017.

\begin{tabular}{|c|c|c|c|c|c|c|c|c|}
\hline & \multicolumn{4}{|c|}{ HIV-Negative/Unknown } & \multicolumn{4}{|c|}{ HIV-Positive } \\
\hline & \multicolumn{4}{|c|}{ Higher Risk Anal Sex (Yes 1457 vs. No 6664) } & \multicolumn{4}{|c|}{ Higher Risk Anal Sex (Yes 1457 vs. No 6664) } \\
\hline & OR & \multicolumn{2}{|c|}{$95 \% \mathrm{CI}$} & $p$ & OR & \multicolumn{2}{|c|}{$95 \% \mathrm{CI}$} & $p$ \\
\hline $16-29$ & Ref & & & & Ref & & & \\
\hline $30-39$ & 1.44 & 1.13 & 1.83 & 0.003 & 0.80 & 0.51 & 1.27 & 0.351 \\
\hline $40+$ & 1.39 & 0.98 & 1.97 & 0.069 & 0.56 & 0.35 & 0.88 & 0.013 \\
\hline \multicolumn{9}{|l|}{ Sexual Orientation } \\
\hline Gay & Ref & & & & Ref & & & \\
\hline Bisexual & 1.39 & 0.99 & 1.96 & 0.056 & 0.73 & 0.52 & 1.03 & 0.071 \\
\hline Other & 1.02 & 0.72 & 1.44 & 0.915 & 1.06 & 0.76 & 1.46 & 0.736 \\
\hline \multicolumn{9}{|l|}{ Ethnicity } \\
\hline White & Ref & & & & Ref & & & \\
\hline Asian & 0.57 & 0.38 & 0.86 & 0.008 & 1.37 & 0.79 & 2.40 & 0.264 \\
\hline Indigenous & 1.20 & 0.66 & 2.20 & 0.545 & 0.94 & 0.48 & 1.85 & 0.861 \\
\hline Latino & 1.16 & 0.69 & 1.96 & 0.577 & 1.26 & 0.51 & 3.10 & 0.611 \\
\hline Other & 0.89 & 0.45 & 1.77 & 0.738 & 3.05 & 1.85 & 5.04 & $<0.0001$ \\
\hline \multicolumn{9}{|l|}{ Born in Canada } \\
\hline No & Ref & & & & Ref & & & \\
\hline Yes & 1.04 & 0.77 & 1.41 & 0.787 & 0.81 & 0.55 & 1.19 & 0.284 \\
\hline \multicolumn{9}{|l|}{ Neighborhood } \\
\hline Downtown/West End & Ref & & & & Ref & & & \\
\hline Elsewhere Vancouver & 0.72 & 0.57 & 0.90 & 0.004 & 1.26 & 0.95 & 1.67 & 0.106 \\
\hline Outside Vancouver & 0.94 & 0.72 & 1.23 & 0.649 & 1.34 & 0.98 & 1.84 & 0.067 \\
\hline \multicolumn{9}{|l|}{ Formal Education } \\
\hline Some or completed high school & Ref & & & & Ref & & & \\
\hline \multicolumn{8}{|l|}{ Annual Income } & 0.066 \\
\hline$<\$ 30,000$ & Ref & & & & Ref & & & \\
\hline at least $\$ 30,000$ & 1.21 & 0.99 & 1.48 & 0.058 & 1.47 & 1.12 & 1.92 & 0.005 \\
\hline \multicolumn{9}{|l|}{ Current Regular Partner } \\
\hline No & Ref & & & & Ref & & & \\
\hline Yes, but not common law/married & 0.93 & 0.76 & 1.13 & 0.452 & 1.29 & 0.97 & 1.71 & 0.085 \\
\hline Yes, common law/married & 0.87 & 0.67 & 1.12 & 0.274 & 1.19 & 0.92 & 1.55 & 0.185 \\
\hline \multicolumn{9}{|l|}{ Usage of PrEP } \\
\hline No & Ref & & & & & & & \\
\hline Yes & 2.20 & 1.41 & 3.42 & 0.001 & & & & \\
\hline
\end{tabular}


Table 2. Cont.

\begin{tabular}{|c|c|c|c|c|c|c|c|c|}
\hline \multirow{4}{*}{$\begin{array}{c} \\
\text { Never heard of PrEP } \\
\text { Escort Work }\end{array}$} & \multirow{2}{*}{\multicolumn{4}{|c|}{$\begin{array}{c}\text { HIV-Negative/Unknown } \\
\text { Higher Risk Anal Sex (Yes } 1457 \text { vs. No 6664) }\end{array}$}} & \multirow{2}{*}{\multicolumn{4}{|c|}{$\begin{array}{c}\text { HIV-Positive } \\
\text { Higher Risk Anal Sex (Yes } 1457 \text { vs. No 6664) }\end{array}$}} \\
\hline & & & & & & & & \\
\hline & \multirow{2}{*}{$\begin{array}{l}\text { OR } \\
0.81\end{array}$} & \multicolumn{2}{|c|}{$95 \% \mathrm{CI}$} & \multirow{2}{*}{$\frac{p}{p}$} & \multirow[t]{2}{*}{ OR } & \multicolumn{2}{|c|}{$95 \%$ CI } & \multirow[t]{2}{*}{$p$} \\
\hline & & 0.66 & 0.98 & & & & & \\
\hline No & Ref & & & & Ref & & & \\
\hline Yes, in P6M & 1.77 & 1.18 & 2.65 & 0.006 & 1.17 & 0.72 & 1.90 & 0.531 \\
\hline Yes, not in P6M & 1.72 & 1.20 & 2.48 & 0.004 & 1.29 & 0.88 & 1.90 & 0.193 \\
\hline \multicolumn{9}{|l|}{ Attended Group Sex P6M } \\
\hline No & Ref & & & & Ref & & & \\
\hline Yes & 1.44 & 1.21 & 1.73 & $<0.0001$ & 1.15 & 0.91 & 1.45 & 0.238 \\
\hline \multicolumn{9}{|l|}{ AUDIT Zone } \\
\hline Low-risk (scores 0 to 7 ) & Ref & & & & Ref & & & \\
\hline Medium-risk (scores 8 to 15 ) & 1.01 & 0.84 & 1.21 & 0.935 & 1.02 & 0.73 & 1.43 & 0.903 \\
\hline Harmful (scores 16 to 19 ) & 1.21 & 0.89 & 1.65 & 0.234 & 0.93 & 0.56 & 1.56 & 0.788 \\
\hline Possible dependence (scores 20 and over) & 1.57 & 1.09 & 2.28 & 0.017 & 0.88 & 0.46 & 1.67 & 0.684 \\
\hline \multicolumn{9}{|l|}{ On ART Ever } \\
\hline No & & & & & Ref & & & \\
\hline Yes & & & & & 2.65 & 0.35 & 19.90 & 0.343 \\
\hline \multicolumn{9}{|l|}{ Treatment Adherence P12M } \\
\hline $95 \%$ or greater & & & & & Ref & & & \\
\hline$<95 \%$ & & & & & 1.15 & 0.90 & 1.48 & 0.266 \\
\hline Never on ART/start within $12 \mathrm{M}$ & & & & & 1.38 & 0.92 & 2.07 & 0.116 \\
\hline Viral Load $<200$ & & & & & & & & \\
\hline No & & & & & Ref & & & \\
\hline Yes & & & & & 0.91 & 0.63 & 1.30 & 0.601 \\
\hline Prevention Strategies & OR & & & $p$ & OR & & & $p$ \\
\hline Always Using Condoms & & & & & & & & \\
\hline No & Ref & & & & Ref & & & \\
\hline Yes & 0.38 & 0.32 & 0.45 & $<0.0001$ & 0.42 & 0.31 & 0.58 & $<0.0001$ \\
\hline Seropositioning & & & & & & & & \\
\hline No & Ref & & & & Ref & & & \\
\hline Yes & 1.69 & 1.39 & 2.06 & $<0.0001$ & 1.77 & 1.41 & 2.23 & $<0.0001$ \\
\hline No Anal Sex & & & & & & & & \\
\hline No & Ref & & & & Ref & & & \\
\hline Yes & 0.76 & 0.65 & 0.90 & 0.001 & 0.77 & 0.63 & 0.95 & 0.013 \\
\hline Serosorting & & & & & & & & \\
\hline No & Ref & & & & Ref & & & \\
\hline Yes & 1.18 & 1.00 & 1.39 & 0.046 & 1.09 & 0.89 & 1.35 & 0.403 \\
\hline Viral-Load Sorting & & & & & & & & \\
\hline No & Ref & & & & Ref & & & \\
\hline Yes & 2.77 & 2.18 & 3.53 & $<0.0001$ & 1.89 & 1.50 & 2.38 & $<0.0001$ \\
\hline Withdrawal & & & & & & & & \\
\hline No & Ref & & & & Ref & & & \\
\hline Yes & 1.08 & 0.90 & 1.30 & 0.410 & 1.44 & 1.14 & 1.81 & 0.002 \\
\hline Asking Status & & & & & & & & \\
\hline No & Ref & & & & Ref & & & \\
\hline Yes & 1.08 & 0.90 & 1.30 & 0.410 & 0.98 & 0.81 & 1.19 & 0.860 \\
\hline Event-Level Variables & OR & & & $p$ & OR & & & $p$ \\
\hline Alcohol & & & & & & & & \\
\hline No & Ref & & & & Ref & & & \\
\hline Yes & 2.26 & 1.82 & 2.82 & $<0.0001$ & 1.11 & 0.89 & 1.40 & 0.346 \\
\hline Cannabis & & & & & & & & \\
\hline No & Ref & & & & Ref & & & \\
\hline Yes & 1.75 & 1.47 & 2.07 & $<0.0001$ & 1.02 & 0.76 & 1.37 & 0.879 \\
\hline Erectile Drugs & & & & & & & & \\
\hline No & Ref & & & & Ref & & & \\
\hline Yes & 1.67 & 1.25 & 2.23 & 0.001 & 1.03 & 0.83 & 1.29 & 0.765 \\
\hline Poppers & & & & & & & & \\
\hline No & Ref & & & & Ref & & & \\
\hline Yes & 2.44 & 1.70 & 3.52 & $<0.0001$ & 1.29 & 1.00 & 1.66 & 0.049 \\
\hline Ecstasy/MDMA & & & & & & & & \\
\hline No & Ref & & & & Ref & & & \\
\hline Yes & 2.59 & 1.77 & 3.79 & $<0.0001$ & 1.17 & 0.67 & 2.06 & 0.583 \\
\hline
\end{tabular}


Table 2. Cont.

\begin{tabular}{|c|c|c|c|c|c|c|c|c|}
\hline & \multicolumn{4}{|c|}{ HIV-Negative/Unknown } & \multicolumn{4}{|c|}{ HIV-Positive } \\
\hline & \multicolumn{4}{|c|}{ Higher Risk Anal Sex (Yes 1457 vs. No 6664) } & \multicolumn{4}{|c|}{ Higher Risk Anal Sex (Yes 1457 vs. No 6664) } \\
\hline & OR & \multicolumn{2}{|c|}{$95 \%$ CI } & $p$ & OR & \multicolumn{2}{|c|}{$95 \%$ CI } & $p$ \\
\hline \multicolumn{9}{|l|}{ GHB } \\
\hline No & Ref & & & & Ref & & & \\
\hline Yes & 2.26 & 1.82 & 2.82 & $<0.0001$ & 0.98 & 0.71 & 1.33 & 0.879 \\
\hline \multicolumn{9}{|l|}{ Crystal Methamphetamine } \\
\hline No & Ref & & & & Ref & & & \\
\hline Yes & 1.75 & 1.47 & 2.07 & $<0.0001$ & 1.06 & 0.80 & 1.40 & 0.696 \\
\hline Continuous Variables & OR & \multicolumn{2}{|c|}{$95 \%$ CI } & $p$ & OR & \multicolumn{2}{|c|}{$95 \%$ CI } & $p$ \\
\hline Male sex number P6M & 1.00 & 1.00 & 1.01 & 0.001 & 1.00 & 1.00 & 1.01 & 0.267 \\
\hline Anal sex number P6M & 1.01 & 1.00 & 1.02 & 0.001 & 1.00 & 1.00 & 1.01 & 0.038 \\
\hline Treatment optimism-skepticism scale & 1.09 & 1.07 & 1.12 & $<0.0001$ & 1.07 & 1.05 & 1.10 & $<0.0001$ \\
\hline Sexual altruism scale (communal) & 0.56 & 0.49 & 0.64 & $<0.0001$ & 0.76 & 0.66 & 0.87 & $<0.0001$ \\
\hline Sexual altruism scale (personal) & 0.54 & 0.47 & 0.63 & $<0.0001$ & 0.77 & 0.65 & 0.90 & 0.001 \\
\hline Sexual sensation seeking scale & 1.12 & 1.09 & 1.16 & $<0.0001$ & 1.09 & 1.05 & 1.13 & $<0.0001$ \\
\hline
\end{tabular}

Notes: P6M = Past six months; P12M = Past 12 months; PrEP = Pre-exposure prophlaxis; ART = Antiretroviral Therapy; AUDIT = Alcohol Use Disorders Identification Test; MDMA = 3,4-methylenedioxymethamphetamine; GHB = 3,4-methylenedioxymethamphetamine.

For trends among HIV-negative/unknown gbMSM, we found that higher risk anal sex events increased over time (first time period prevalence: $13 \%$, last time period prevalence: $29 \%)(\mathrm{OR}=1.006 ; 95 \% \mathrm{CI}=1.002,1.011, p=0.009)$. We found event-level CAS increased over the study period $(\mathrm{OR}=1.015 ; 95 \% \mathrm{CI}=1.011,1.019, p<0.001)$, anal sex with HIV-negative partners increased over the study period $(\mathrm{OR}=1.013 ; 95 \% \mathrm{CI}=1.009,1.017, p<0.001)$, and anal sex with HIV-positive gbMSM increased over the study period ( $\mathrm{OR}=1.012$; $95 \% \mathrm{CI}=1.006,1.017, p=0.000$ ). In relation to event-level substance use among HIVnegative/unknown gbMSM over the study period, event-level alcohol use decreased (first time period prevalence: $46 \%$, last time period prevalence: $27 \%)(\mathrm{OR}=0.989 ; 95 \% \mathrm{CI}=0.985$, $0.993, p<0.001$ ), and erectile drug use increased (first time period prevalence: $6 \%$, last time period prevalence: $9 \%)(\mathrm{OR}=1.010 ; 95 \% \mathrm{CI}=1.001,1.020, p=0.031)$. Full results can be found in Figure 1.

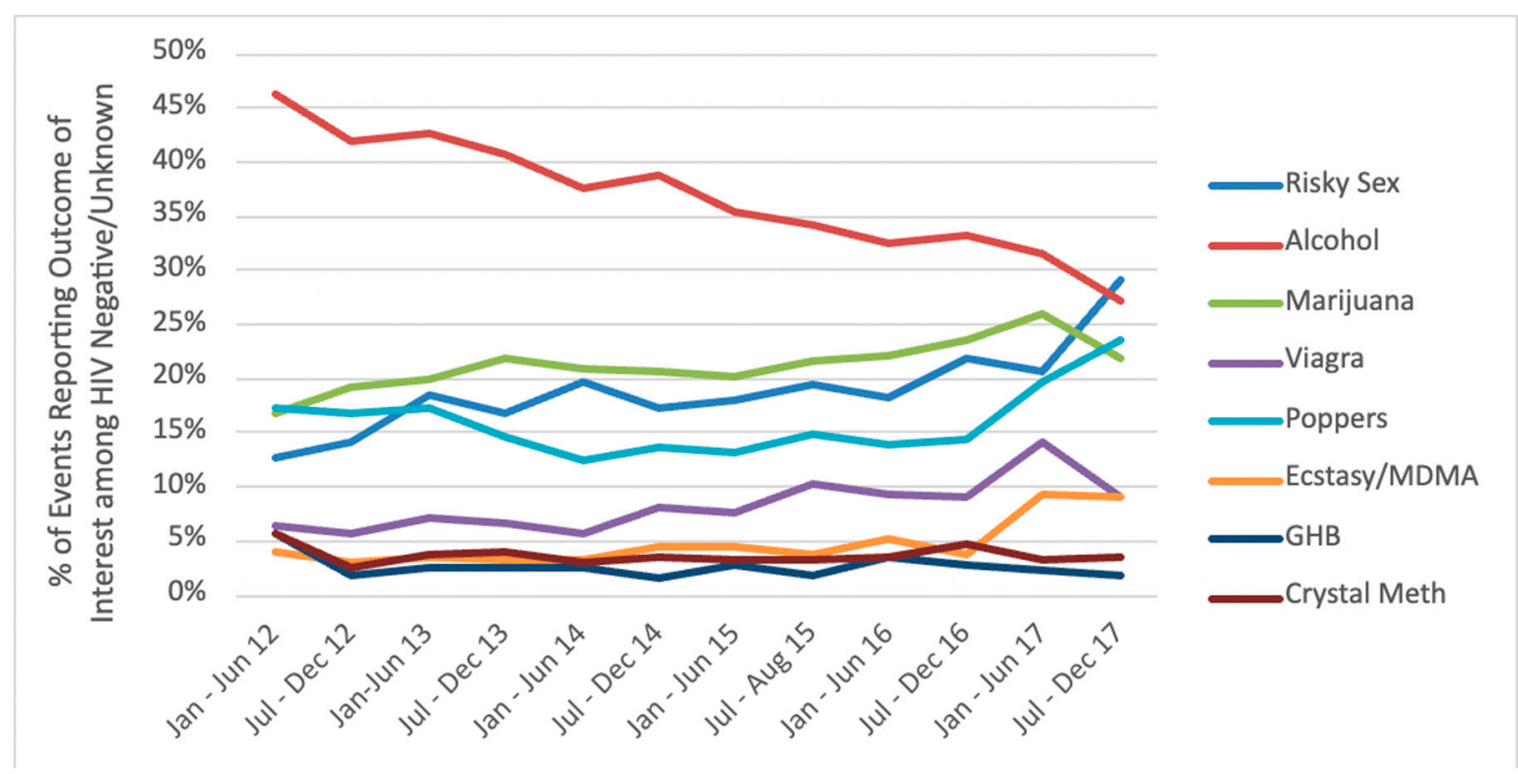

Figure 1. Percentage trends of sexual events reporting substance use and higher risk anal sex among HIVnegative/unknown gbMSM in Metro Vancouver. Notes: MDMA = 3,4-methylenedioxymethamphetamine; GHB = 3,4methylenedioxymethamphetamine. 
For trends among HIV-positive gbMSM, our model found higher risk anal sex increased over time (first time period prevalence: $16 \%$, last time period prevalence: $38 \%$ ) $(\mathrm{OR}=1.006 ; 95 \% \mathrm{CI}=1.001,1.012, p=0.025)$. In relation to event-level substance use, we found that popper use decreased over time (first time period prevalence: $38 \%$, last time period prevalence: $31 \%)(\mathrm{OR}=0.991 ; 95 \% \mathrm{CI}=0.984,0.998, p=0.007)$. Full results can be found in Figure 2.

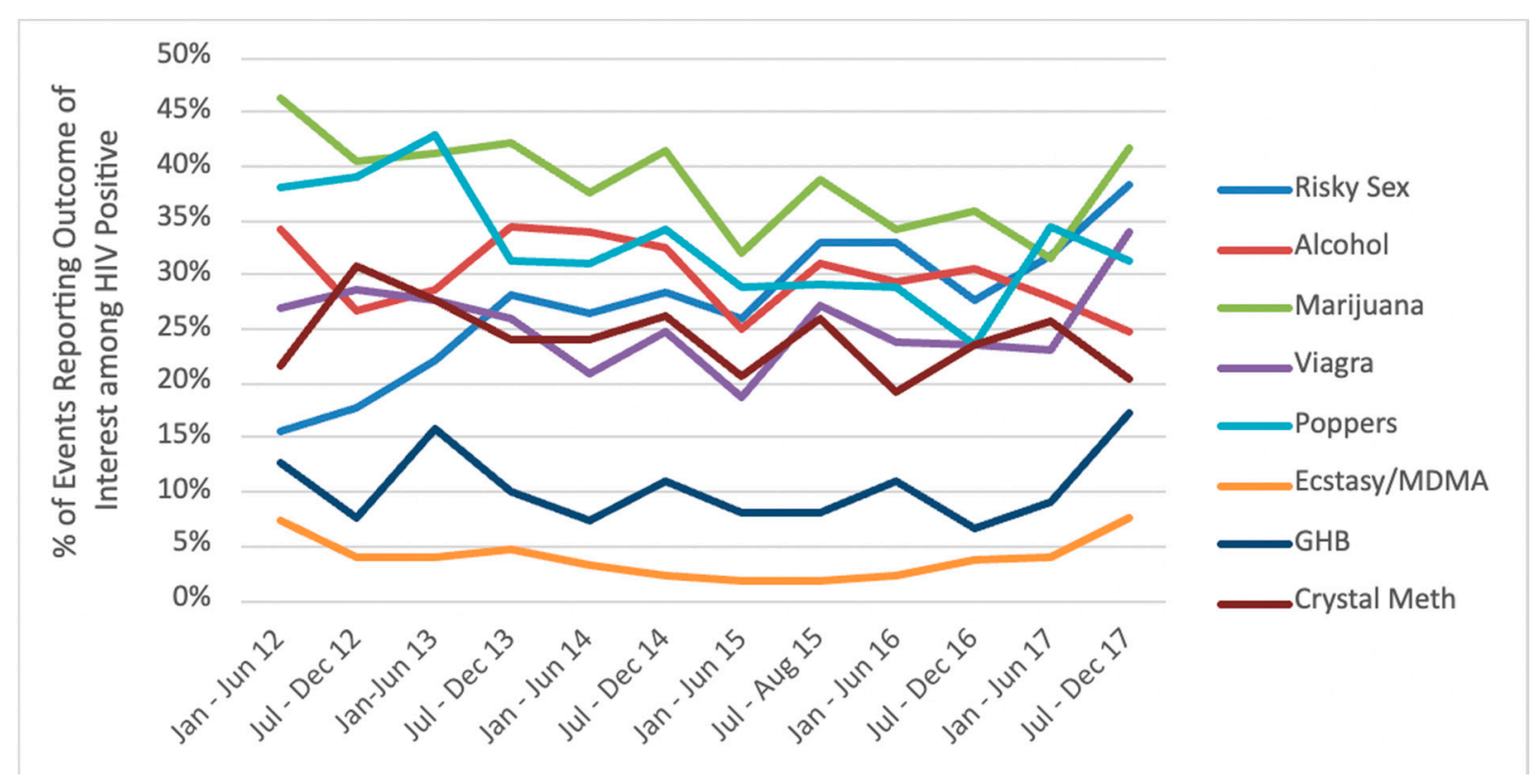

Figure 2. Percentage trends of sexual events reporting substance use and higher risk anal sex among HIV-positive gbMSM in Metro Vancouver. Notes: MDMA = 3,4-methylenedioxymethamphetamine; GHB = 3,4-methylenedioxymethamphetamine.

We did not find any significant interactions between time and substance use on the likelihood of event-level higher-risk anal sex. However, we did find a number of timespecific significant interactions. Full results can be found in Table 3.

We conducted a post hoc lost to follow-up analysis and found that participants LTFU reported less higher-risk anal sex among HIV-negative/unknown gbMSM and more ecstasy/MDMA use among HIV-positive gbMSM.

Table 3. Univariable temporal trends and interactions of substance use and higher risk anal sex prevalence among gbMSM in Metro Vancouver, 2012-2017.

\begin{tabular}{ccccccccc}
\hline Trend & \multicolumn{3}{c}{ HIV-Negative/Unknown } & \multicolumn{4}{c}{ HIV-Positive } \\
\hline & OR & \multicolumn{9}{c}{$\mathbf{9 5 \%}$ CI } & $\boldsymbol{p}$ & OR & $\mathbf{9 5 \%}$ CI & $\boldsymbol{p}$ \\
\hline Higher risk anal sex & 1.006 & 1.002 & 1.011 & 0.009 & 1.006 & 1.001 & 1.012 & 0.025 \\
Condomless anal sex & 1.015 & 1.011 & 1.019 & $<0.0001$ & 1.002 & 0.997 & 1.007 & 0.389 \\
Knew neg vs. unknown & 1.013 & 1.009 & 1.017 & $<0.0001$ & 1.005 & 0.999 & 1.012 & 0.104 \\
Knew pos vs. unknown & 1.012 & 1.006 & 1.018 & 0.000 & 0.998 & 0.992 & 1.004 & 0.495 \\
Alcohol & 0.989 & 0.985 & 0.993 & $<0.0001$ & 0.996 & 0.990 & 1.002 & 0.207 \\
Cannabis & 1.004 & 0.999 & 1.010 & 0.146 & 0.995 & 0.989 & 1.001 & 0.095 \\
Erectile drugs & 1.010 & 1.001 & 1.020 & 0.031 & 0.999 & 0.992 & 1.007 & 0.866 \\
Poppers & 0.998 & 0.990 & 1.005 & 0.561 & 0.991 & 0.984 & 0.998 & 0.007 \\
Ecstasy/MDMA & 1.012 & 1.000 & 1.024 & 0.053 & 0.997 & 0.976 & 1.018 & 0.760 \\
GHB & 1.000 & 0.986 & 1.015 & 0.952 & 1.001 & 0.990 & 1.012 & 0.869 \\
\hline
\end{tabular}


Table 3. Cont.

\begin{tabular}{|c|c|c|c|c|c|c|c|c|}
\hline \multirow[b]{3}{*}{ Crystal methamphetamine } & \multicolumn{4}{|c|}{ HIV-Negative/Unknown } & \multicolumn{4}{|c|}{ HIV-Positive } \\
\hline & \multirow{2}{*}{$\begin{array}{c}\text { OR } \\
0.997\end{array}$} & \multicolumn{2}{|c|}{$95 \% \mathrm{CI}$} & \multirow{2}{*}{$\begin{array}{c}p \\
0.705\end{array}$} & \multirow{2}{*}{$\begin{array}{c}\text { OR } \\
0.998\end{array}$} & \multicolumn{2}{|c|}{$95 \%$ CI } & \multirow{2}{*}{$\begin{array}{c}p \\
0.649\end{array}$} \\
\hline & & 0.984 & 1.011 & & & 0.991 & 1.006 & \\
\hline \multicolumn{9}{|l|}{ Interactions } \\
\hline & OR & \multicolumn{2}{|c|}{$95 \% \mathrm{CI}$} & $p$ & OR & \multicolumn{2}{|c|}{$95 \%$ CI } & $p$ \\
\hline Alcohol $X$ trend & 1.002 & 0.994 & 1.010 & 0.580 & 0.994 & 0.985 & 1.004 & 0.248 \\
\hline 01/2012: User vs. not & 1.051 & 0.778 & 1.419 & 0.746 & 1.372 & 0.942 & 1.997 & 0.099 \\
\hline 12/2017: User vs. not & 1.228 & 0.891 & 1.694 & 0.210 & 0.918 & 0.591 & 1.424 & 0.701 \\
\hline Cannabis $\mathrm{X}$ trend & 1.004 & 0.995 & 1.013 & 0.410 & 1.004 & 0.995 & 1.014 & 0.358 \\
\hline 01/2012: User vs. not & 0.958 & 0.664 & 1.381 & 0.817 & 0.868 & 0.560 & 1.345 & 0.527 \\
\hline 12/2017: User vs. not & 1.267 & 0.855 & 1.878 & 0.239 & 1.188 & 0.761 & 1.856 & 0.448 \\
\hline Erectile drugs $X$ trend & 1.007 & 0.994 & 1.020 & 0.319 & 0.997 & 0.987 & 1.008 & 0.617 \\
\hline 01/2012: User vs. not & 1.797 & 1.100 & 2.936 & 0.019 & 1.145 & 0.715 & 1.833 & 0.574 \\
\hline 12/2017: User vs. not & 2.890 & 1.679 & 4.975 & 0.000 & 0.942 & 0.626 & 1.419 & 0.776 \\
\hline Poppers X trend & 1.000 & 0.991 & 1.010 & 0.971 & 1.001 & 0.989 & 1.012 & 0.905 \\
\hline 01/2012: User vs. not & 1.737 & 1.254 & 2.404 & 0.001 & 1.258 & 0.797 & 1.987 & 0.325 \\
\hline 12/2017: User vs. not & 1.759 & 1.137 & 2.720 & 0.011 & 1.322 & 0.803 & 2.178 & 0.273 \\
\hline Ecstasy/MDMA X trend & 1.012 & 0.993 & 1.031 & 0.226 & 0.981 & 0.962 & 1.000 & 0.054 \\
\hline 01/2012: User vs. not & 1.109 & 0.560 & 2.198 & 0.767 & 2.229 & 0.941 & 5.280 & 0.069 \\
\hline 12/2017: User vs. not & 2.543 & 1.171 & 5.523 & 0.018 & 0.568 & 0.250 & 1.287 & 0.175 \\
\hline GHB $X$ trend & 0.989 & 0.966 & 1.013 & 0.360 & 0.992 & 0.977 & 1.007 & 0.293 \\
\hline 01/2012: User vs. not & 3.506 & 1.680 & 7.317 & 0.001 & 1.315 & 0.639 & 2.705 & 0.457 \\
\hline 12/2017: User vs. not & 1.610 & 0.544 & 4.770 & 0.390 & 0.735 & 0.440 & 1.227 & 0.240 \\
\hline Crystal methamphetamine $X$ trend & 0.978 & 0.957 & 1.000 & 0.054 & 0.994 & 0.982 & 1.006 & 0.312 \\
\hline 01/2012: User vs. not & 5.048 & 2.376 & 10.726 & $<0.0001$ & 1.324 & 0.903 & 1.941 & 0.150 \\
\hline 12/2017: User vs. not & 1.065 & 0.391 & 2.900 & 0.901 & 0.845 & 0.449 & 1.592 & 0.602 \\
\hline
\end{tabular}

\section{Discussion}

Our research explored temporal trends and associations between substance use and higher risk sex during anal sex events among gbMSM in Vancouver, BC. We found that event-level sexual risk increased over time for both HIV-negative/unknown gbMSM and HIV-positive gbMSM over nearly six years of follow-up. However, HIV-negative/unknown gbMSM reported more frequent CAS with serodifferent or unknown status partners compared to HIV-positive gbMSM. Although we did not find longitudinally significant interactions between substance use and time on higher-risk anal sex, we found a number of time-specific associations that warrant further exploration.

We found event-level higher risk anal sex increased over time for both HIV-negative/ unknown gbMSM and HIV-positive gbMSM. Among HIV-negative/unknown gbMSM, we also found increasing trends of overall CAS. Trends of gbMSM gaining regular partnerships and fewer sex partners may better explain this finding [26]. As gbMSM are more likely to have a regular partner, they may also be less likely to wear condoms with their regular partner over time [27]. Additionally, we found increasing trends of CAS with known HIVnegative versus unknown partners and CAS with known HIV-positive versus unknown partners. We hypothesize that based on increasing trends of HIV testing across Canada [28], gbMSM are also reporting fewer unknown HIV status partners over time.

Exploring substance use trends among HIV-negative/unknown gbMSM, we found alcohol use decreased and erectile drugs increased over time. The cohort aging effect may explain decreases in alcohol as findings indicate alcohol consumption and use of illicit substances tend to decrease across the lifespan [29]. In relation to erectile drug use, evidence suggests erectile drugs are commonly used by gbMSM, who are more sexually active and who use erectile drugs specifically to enhance sexual performance and duration [30]. The longitudinal nature and aging cohort effect may further explain increases in erectile drug use over time. However, we did not find these trends among HIV-positive gbMSM. Instead, we found popper use decreased over time among HIVpositive gbMSM. This finding is significant because, in 2013, Canada banned the sale of poppers, which may have precipitated this decline. Although the sale of poppers has been banned, critics argue that this ban is not supported by science and that this 
has led to an unregulated market, increasing the risk of dangerous off-market products and forcing access to poppers in potentially dangerous ways [31]. A recent analysis on popper use among young gbMSM found high lifetime and recent popper use, yet dependency symptoms and risky consumption or problems arising from using poppers were low [32]. Overall, differences in substance use trends among gbMSM by serostatus have been identified elsewhere, yet further research is needed to delineate unique eventlevel substance use factors by serostatus [33].

We identified noteworthy findings from our univariable analyses. First, our findings for PrEP usage should be interpreted with caution given the limited number of gbMSM reporting ever using PrEP, which was less than $2 \%$ of participants reporting using PrEP at any point in our study. Recently, in British Columbia, PrEP became publicly funded and freely available in January 2018 [34]. Thus, our analysis provides a basis for further exploration of PrEP use, substance use and sexual risk as PrEP use increases. It is important to note that biomedical interventions, such as PrEP, are changing gbMSM's notions of "safe" and "risky" sex, as CAS with a serodiscordant or unknown partner may not place increased HIV risk to individuals on PrEP. However, also important to consider is that biomedical interventions only protect against HIV, and STI risk may still be associated with CAS. Thus, condoms still provide relevant protection and should be paired with health promotion and programming that embraces a broader STBBI framework. Second, we did not find a significant difference between viral-load status and higher risk anal sex, which is inconsistent with previous findings [35]. We hypothesize that these differences may be explained by the low proportion of gbMSM with a detectable viral load in our study. At baseline, only $16 \%$ of HIV-positive gbMSM reported a viral load of $\geq 200$, and this rate is expected to have gone down as TasP scale-up increased. Third, our results found greater endorsement of HIV treatment optimism was associated with higher odds of sexual risk. Previous research exploring HIV treatment optimism among gbMSM found increasing trends of HIV-optimism over time, but no longitudinal differences in higher risk anal sex for both HIV-negative and HIV-positive men [36]. Differences may be explained by our focus on event-level sexual risk and variations in sexual behaviors with specific partners versus overall sexual risk behaviors.

We did not find statistically significant interactions between time and substance use on higher-risk anal sex. However, we found that erectile drug use and poppers use were consistently associated with higher risk anal sex throughout the study period for HIVnegative/unknown gbMSM. These findings are consistent with previous literature, which demonstrates individual associations between erectile drug use and popper use on sexual risk $[37,38]$. Interestingly, we found GHB, crystal methamphetamine, and ecstasy/MDMA use associated with sexual risk at one point in our study (baseline or end of study), but the associations were not maintained throughout the study period. We hypothesize that interactions between substance use and sexual risk over time were not significant because rates of substances may have varied over time with different partners. As such, gbMSM, who engage in multiple sex partners, may have had significant sexual risk at one point in our study, but sexual partners and substances used may have changed over time. Alternatively, gbMSM may have had fewer sexual partners over time, whereby sexual risk and substance use decreased with time and partnership.

This research is subject to limitations. First, although our research used sexual eventlevel data, these are likely not representative of all sexual events between partners and are subject to recall bias. Still, our focus on event-level data is advantageous in comparison to cross-sectional data to causally examine the relationship between substance use and sexual risk. Second, our findings may not be comparable outside of Vancouver, British Columbia, especially given the TasP context and promotion in the city. Third, we did not distinguish between different partner types (e.g., casual or main partners), which may influence the use of substances, sexual behaviors, and the relationship between these. Fourth, we could not distinguish between medically prescribed erectile drugs and nonmedically prescribed. However, the prevalence of recreational erectile drug use among 
gbMSM is high, and erectile drug use (regardless of the prescription) has been identified as an important indicator of sexual risk in existing literature [39,40]. Fifth, many of our significant findings for substance use and higher risk anal sex diminished over time. We theorized this might be due to the LTFU of high-risk/high substance-using participants and completed post hoc analyses to assess this. We found two significant differences for participants LTFU, and these findings may limit the scope of interpretation for these results.

\section{Conclusions}

Our findings indicate that sexual risk is associated with event-level substance use, behavioral and psychosocial factors. These findings provide a more comprehensive understanding of the effects of event-level substance use on sexual risk among Canadian gbMSM through longitudinal follow-up. Moreover, future interventions must address increasing rates of sexual risk through continued education on STIs, increased access to safer sex materials, and continued preventative screening. Understanding the nuances of individual substances as well as polysubstance use will be beneficial in developing targeted interventions for substance-using gbMSM in managing sexual risk. By focusing on separate sexualized events with different partners and substances used, sexual risk programs can target strategies for reducing risk in certain scenarios and how individuals can apply this knowledge to future sexual events.

Author Contributions: Conceptualization, N.J.L., J.M.S.; Data curation, P.S.; Formal analysis, Z.C.; Project administration, G.O., A.L.; Supervision, N.J.L., D.M.M.; Writing-original draft, J.M.S.; Writing-review \& editing, G.O., H.L.A., A.L., K.G.C., E.A.R., D.M.M.; Funding acquisition, R.S.H. All authors have read and agreed to the published version of the manuscript.

Funding: This work was supported by the National Institute on Drug Abuse (R01DA031055-01A1) and the Canadian Institutes of Health Research (MOP-107544, FND-143342, PJT-153139). N.J.L. was supported by a CANFAR/CTN Postdoctoral Fellowship Award. H.L.A. was supported by a Postdoctoral Fellowship award from the Canadian Institutes of Health Research (grant \#MFE-152443). D.M.M. and N.J.L. are supported by Michael Smith Foundation for Health Research Scholar Awards (\#5209, \#16863). K.G.C. is supported by a Canadian Institutes of Health Research Health Systems Impact Fellowship award, a Michael Smith Foundation for Health Research Trainee Award, and a Canadian HIV Trials Network/Canadian Foundation for AIDS Research Postdoctoral Fellowship award. The authors of this study have no conflicts of interest to disclose. All authors have read and agreed to the published version of the manuscript.

Institutional Review Board Statement: The study was conducted according to the guidelines of the Declaration of Helsinki and approved by the Institutional Review Board of The University of British Columbia, Simon Fraser University and the University of Victoria (H11-00691, Approved 15 April 2019).

Informed Consent Statement: Informed consent was obtained from all subjects involved in the study.

Data Availability Statement: Not Applicable.

Acknowledgments: We would like to thank the Momentum Health Study participants, office staff and community advisory board, as well as our community partners: Health Initiative for Men, YouthCO HIV and Hep C Society, and Positive Living Society of BC.

Conflicts of Interest: The authors declare no conflict of interest.

\section{References}

1. Public Health Agency of Canada. Report on Sexually Transmitted Infections in Canada, 2017; Government of Canada: Ottawa, ON, Cancada, 2019.

2. Ward, H.; Rönn, M. Contribution of sexually transmitted infections to the sexual transmission of HIV. Curr. Opin. HIV AIDS 2010, 5, 305-310. [CrossRef] [PubMed]

3. Cassels, S.; Katz, D.A. Seroadaptation among Men Who Have Sex with Men: Emerging Research Themes. Curr. HIV/AIDS Rep. 2013, 10, 305-313. [CrossRef] [PubMed] 
4. Shuper, P.A.; MacLachlan, D.J.; Joharchi, N.; Guimond, T.H.; Maxwell, J.; Adam, B.D. HIV Risk and Protective Factors in the Context of Alcohol and Substance Use During Pride. AIDS Behav. 2018, 22, 2797-2806. [CrossRef] [PubMed]

5. Wayal, S.; Hart, G.; Copas, A.; Edwards, S.; Cassell, J. O18 7Sexual behaviour, partnership patterns and STI diagnoses among HIV positive MSM: Implications for HIV/STIs transmission and partner notification. Sex. Transm. Infect. 2012, 88 (Suppl. 1), A7.

6. Grewal, R.; Allen, V.G.; Gardner, S.; Moravan, V.; Tan, D.H.S.; Raboud, J.; Bayoumi, A.M.; Kaul, R.; Mazzulli, T.; McGee, F.; et al. Serosorting and recreational drug use are risk factors for diagnosis of genital infection with chlamydia and gonorrhoea among HIV-positive men who have sex with men: Results from a clinical cohort in Ontario, Canada. Sex. Transm. Infect. 2017, 93, 71-75. [CrossRef]

7. Card, K.G.; Armstrong, H.L.; Carter, A.; Cui, Z.; Wang, C.; Zhu, J.; Lachowsky, N.J.; Moore, D.M.; Hogg, R.S.; Roth, E.A. Assessing the longitudinal stability of latent classes of substance use among gay, bisexual, and other men who have sex with men. Drug Alcohol Depend. 2018, 188, 348-355. [CrossRef] [PubMed]

8. Lafortune, D.; Blais, M.; Miller, G.; Dion, L.; Lalonde, F.; Dargis, L. Psychological and Interpersonal Factors Associated with Sexualized Drug Use Among Men Who Have Sex with Men: A Mixed-Methods Systematic Review. Arch. Sex. Behav. 2021, 50, 427-460. [CrossRef] [PubMed]

9. McCall, H.; Adams, N.; Mason, D.V.; Willis, J. What is chemsex and why does it matter? BMJ Br. Med J. 2015, 351, h5790. [CrossRef] [PubMed]

10. González-Baeza, A.; Dolengevich-Segal, H.; Pérez-Valero, I.; Cabello, A.; Téllez, M.J.; Sanz, J.; Pérez-Latorre, L.; Bernardino, J.I.; Troya, J.; De La Fuente, S.; et al. Sexualized Drug Use (Chemsex) Is Associated with High-Risk Sexual Behaviors and Sexually Transmitted Infections in HIV-Positive Men Who Have Sex with Men: Data from the U-SEX GESIDA 9416 Study. AIDS Patient Care STDS 2018, 32, 112-118. [CrossRef] [PubMed]

11. Pufall, E.L.; Kall, M.; Shahmanesh, M.; Nardone, A.; Gilson, R.; Delpech, V.; Ward, H.; Positive Voices study group. Sexualized drug use ('chemsex') and high-risk sexual behaviours in HIV-Positive men who have sex with men. HIV Med. 2018, 19, 261-270. [CrossRef]

12. Sewell, J.; Miltz, A.; Lampe, F.C.; Cambiano, V.; Speakman, A.; Phillips, A.N.; Stuart, D.; Gilson, R.; Asboe, D.; Nwokolo, N.; et al. Poly drug use, chemsex drug use, and associations with sexual risk behaviour in HIV-negative men who have sex with men attending sexual health clinics. Int. J. Drug Policy 2017, 43, 33-43. [CrossRef] [PubMed]

13. Bourne, A.; Reid, D.; Hickson, F.; Torres Rueda, S.; Weatherburn, P. Executive Summary-The Chemsex Study: Drug Use in Sexual Settings Among Gay and Bisexual Men in LS; Sigma Research; London School of Hygiene \& Tropical Medicine: London, UK, 2014.

14. Lachowsky, N.J.; Card, K.G.; Cui, Z.; Sereda, P.; Roth, E.A.; Hogg, R.S.; Moore, D.M. Agreement between gay, bisexual and other men who have sex with men's period prevalence and event-level recall of sexual behaviour: An observational respondent-driven sampling study. Sex. Health 2019, 16, 84. [CrossRef] [PubMed]

15. Vosburgh, H.W.; Mansergh, G.; Sullivan, P.S.; Purcell, D.W. A Review of the Literature on Event-Level Substance Use and Sexual Risk Behavior Among Men Who Have Sex with Men. AIDS Behav. 2012, 16, 1394-1410. [CrossRef] [PubMed]

16. Rendina, H.J.; Moody, R.L.; Ventuneac, A.; Grov, C.; Parsons, J.T. Aggregate and event-level associations between substance use and sexual behavior among gay and bisexual men: Comparing retrospective and prospective data. Drug Alcohol Depend. 2015, 154, 199-207. [CrossRef] [PubMed]

17. Sullivan, M.C.; Cruess, D.G.; Huedo-Medina, T.B.; Kalichman, S.C. Substance Use, HIV Serostatus Disclosure, and Sexual Risk Behavior in People Living with HIV: An Event-Level Analysis. Arch. Sex. Behav. 2020, 49, 2005-2018. [CrossRef] [PubMed]

18. Feinstein, B.A.; Newcomb, M.E. Event-Level Associations Among Drinking Motives, Alcohol Consumption, and Condomless Anal Sex in a Sample of Young Men Who Have Sex With Men. AIDS Behav. 2017, 21, 1904-1913. [CrossRef]

19. Melendez-Torres, G.J.; Hickson, F.; Reid, D.; Weatherburn, P.; Bonell, C. Nested Event-Level Case-Control Study of Drug Use and Sexual Outcomes in Multipartner Encounters Reported by Men Who Have Sex with Men. AIDS Behav. 2016, $20,646-654$. [CrossRef] [PubMed]

20. Montaner, J.S.; Lima, V.D.; Harrigan, P.R.; Lourenço, L.; Yip, B.; Nosyk, B.; Wood, E.; Kerr, T.; Shannon, K.; Moore, D.; et al. Expansion of HAART coverage is associated with sustained decreases in HIV/AIDS morbidity, mortality and HIV transmission: The "HIV Treatment as Prevention" experience in a Canadian setting. PLoS ONE 2014, 9, e87872. [CrossRef] [PubMed]

21. Lachowsky, N.J.; Lal, A.; I Forrest, J.; Card, K.G.; Cui, Z.; Sereda, P.; Rich, A.; Raymond, H.F.; A Roth, E.; Moore, D.M.; et al. Including Online-Recruited Seeds: A Respondent-Driven Sample of Men Who Have Sex With Men. J. Med. Internet Res. 2016, 18, e51. [CrossRef] [PubMed]

22. Van De Ven, P.; Crawford, J.; Kippax, S.; Knox, S.; Prestage, G. A scale of optimism-scepticism in the context of HIV treatments. AIDS Care 2000, 12, 171-176. [PubMed]

23. Kalichman, S.C.; Rompa, D. Sexual sensation seeking and Sexual Compulsivity Scales: Reliability, validity, and predicting HIV risk behavior. J. Personal. Assess. 1995, 65, 586. [CrossRef] [PubMed]

24. Nimmons, D.; Folkman, S. Other-Sensitive Motivation for Safer Sex Among Gay Men: Expanding Paradigms for HIV Prevention. AIDS Behav. 1999, 3, 313-324. [CrossRef]

25. Dawson, D.A.; Grant, B.F.; Stinson, F.S.; Zhou, Y. Effectiveness of the Derived Alcohol Use Disorders Identification Test (AUDIT-C) in Screening for Alcohol Use Disorders and Risk Drinking in the US General Population. Alcohol. Clin. Exp. Res. 2005, 29, 844-854. [CrossRef] [PubMed] 
26. Hunt, G.; Wang, L.; Bacani, N.; Card, K.; Sereda, P.; Lachowsky, N.; Roth, E.; Hogg, R.; Moore, D.; Armstrong, H. Generational differences in sexual behaviour and partnering among gay, bisexual, and other men who have sex with men. Can. J. Hum. Sex. 2019, 28, 215-225. [CrossRef]

27. Lachowsky, N.J.; E Dewey, C.; Dickson, N.P.; Saxton, P.J.W.; Hughes, A.J.; Milhausen, R.R.; Summerlee, A.J.S. Habitual condom use across partner type and sexual position among younger gay and bisexual men: Findings from New Zealand HIV behavioural surveillance 2006-2011. Sex. Transm. Infect. 2015, 91, 445-450. [CrossRef] [PubMed]

28. Haddad, N.; Robert, A.; Weeks, A.; Popovic, N.; Siu, W.; Archibald, C. HIV in Canada-Surveillance Report, 2018. Can. Commun. Dis. Rep. 2019, 45, 304-312. [CrossRef] [PubMed]

29. Green, K.E.; Feinstein, B.A. Substance use in lesbian, gay, and bisexual populations: An update on empirical research and implications for treatment. Psychol. Addict. Behav. 2012, 26, 265-278. [CrossRef] [PubMed]

30. Prestage, G.; Jin, F.; Bavinton, B.; Grulich, A.; Brown, G.; Pitts, M.; Hurley, M. Australian Gay and Bisexual Men's Use of Erectile Dysfunction Medications During Recent Sexual Encounters. J. Sex. Med. 2014, 11, 809-819. [CrossRef]

31. Schwartz, C. Vapour and Mirrors: Poppers in the Context of Canadian Drug Policy. 2019. Available online: https://www.cbrc net/vapour_and_mirrors_poppers_in_the_context_of_canadian_drug_policy (accessed on 9 November 2020).

32. Demant, D.; Oviedo-Trespalacios, O. Harmless? A hierarchical analysis of poppers use correlates among young gay and bisexual men. Drug Alcohol Rev. 2019, 38, 465-472. [CrossRef] [PubMed]

33. Sanchez, T.H.; Zlotorzynska, M.; Sineath, R.C.; Kahle, E.; Tregear, S.; Sullivan, P.S. National Trends in Sexual Behavior, Substance Use and HIV Testing Among United States Men Who have Sex with Men Recruited Online, 2013 through 2017. AIDS Behav. 2018, 22, 2413-2425. [CrossRef]

34. British Columbia Centre for Excellence in HIV/AIDS. Spring ARV Update: Publicly-funded PrEP off to strong start in BC. 2018. Available online: http://www.cfenet.ubc.ca/blog/spring-arv-update-publicly-funded-prep-strong-start-bc (accessed on 16 November 2020).

35. Van Kesteren, N.M.C.; Hospers, H.J.; Kok, G. Sexual risk behavior among HIV-positive men who have sex with men: A literature review. Patient Educ. Couns. 2006, 65, 5-20. [CrossRef] [PubMed]

36. Moore, D.M.; Cui, Z.; Lachowsky, N.J.; Rich, A.J.; Roth, E.A.; Raymond, H.F.; Sereda, P.; Montaner, J.; Wong, J.; Armstrong, H.L.; et al. Increasing HIV Treatment Optimism but No Changes in HIV Risk Behavior Among Men Who Have Sex with Men in Vancouver, Canada. J. Acquir. Immune Defic. Syndr. 2017, 76, e98-e101. [CrossRef] [PubMed]

37. Drumright, L.N.; Little, S.J.; Strathdee, S.A.; Slymen, D.J.; Araneta, M.R.G.; Malcarne, V.L.; Daar, E.S.; Gorbach, P.M. Unprotected Anal Intercourse and Substance Use Among Men Who Have Sex With Men With Recent HIV Infection. JAIDS J. Acquir. Immune Defic. Syndr. 2006, 43, 344-350. [CrossRef] [PubMed]

38. Santos, G.-M.; Coffin, P.O.; Das, M.; Matheson, T.; Demicco, E.; Raiford, J.L.; Vittinghoff, E.; Dilley, J.W.; Colfax, G.; Herbst, J.H. Dose-Response Associations Between Number and Frequency of Substance Use and High-Risk Sexual Behaviors Among HIV-Negative Substance-Using Men Who Have Sex With Men (SUMSM) in San Francisco. JAIDS J. Acquir. Immune Defic. Syndr. 2013, 63, 540-544. [CrossRef]

39. Chan, W.L.; Wood, D.M.; Dargan, P.I. Significant Misuse of Sildenafil in London Nightclubs. Substance Use Misuse 2015, 50, 1390-1394. [CrossRef] [PubMed]

40. Fisher, D.G.; Reynolds, G.L.; Ware, M.R.; Napper, L.E. Methamphetamine and Viagra Use: Relationship to Sexual Risk Behaviors. Arch. Sex. Behav. 2011, 40, 273-279. [CrossRef] [PubMed] 